\title{
Phenytoin Cream Reduces Pain and Improves Sleep in Patients Suffering from Neuropathic Pain
}

\section{Keppel Hesselink J M* and Kopsky DJ \\ Institute Neuropathic Pain, Amsterdam, Netherlands \\ *Correspondling author: Keppel HesselinkJ M, Institute Neuropathic Pain, Amsterdam, Netherlands}

Received: October 11, 2017; Accepted: November 01, 2017; Published: November 08, 2017

\begin{abstract}
Patients suffering from neuropathic pain very often have impaired sleep patterns, especially during night. This negatively influences the quality of life and can also result in feelings of depression and/or anxiety. These emotional states often lead to the aggravation of pain, and a vicious circle emerges. We present four patients, all suffering from neuropathic pain due to different causes and interfering considerably with their sleep quality and quality of life. In all four cases after topical phenytoin cream was applied, the pain diminished considerably and sleep quality improved leading to a better quality of life without any adverse events.
\end{abstract}

Keywords: Pain; Phenytoin Cream; Analgesic

\section{Introduction}

In 2014 we started collecting data on the efficacy and safety of topically administered phenytoin cream in patients suffering from neuropathic pain. Phenytoin was introduced in the clinic in 1938 and in addition to the main indication, epilepsy, it has been repositioned in a great number of indications, neuropathic pain being the most recent one (Figure 1) [1,2]. The compound has a number of mechanisms of actions and acts as a broad channel blocking agent especially for many subtypes of sodium channels [3]. Moreover, there are a number of indicators for phenytoin as a neuroprotective agent [4].

To date, we documented in detail the results from 70 patients, most of which were diagnosed as neuropathy due to diabetes mellitus type II, Chronic Idiopathic Axonal Polyneuropathy (CIAP) or chemotherapy-induced peripheral neuropathy (CIPN). Already we have documented in several case reports the safety and efficacy of topical phenytoin cream for the treatment of individual cases with neuropathic pain [5-8].

Some of these patients were identified as responders to topical phenytoin cream via a fast responder test, as outlined in detail elsewhere [7]. Most of our patients suffering from neuropathic pain also complained about sleep problems, and this has a clear negative impact on the quality of life (QoL). Anxiety and depression in these patients are symptoms that further negatively influence the QoL [9]. Such problems seem to occur very often in these patients $[10,11]$. This was a reason to inquire in more detail each patient's experiences on the level of sleep, while being treated with our topical phenytoin formulation.

\section{Case Presentation}

\section{Case 1: Canal stenosis}

For the last 6 years, this 63-year-old male patient has suffered from neuropathic pain in his left big toe, possibly due to the Verbiest syndrome (canal stenosis L5/S1). In 1980 he suffered from the Guillain-Barré syndrome and after recovery his feet remained numb. The main pain location was the left big toe and the pain characteristic was mostly of a stabbing nature, with the patient scoring his pain intensity as 8.5 on the NRS.

Previous analgesic use was pregabalin $75 \mathrm{mg}$ three times daily which was stopped due to side effects (anxiousness, depression, apathy, sweating). The pain was so bothersome that he could not sleep during the night. The use of baclofen $5 \%$ cream resulted in aggravation of pain for the first 10 minutes after application and then the pain vanished completely (from 8.5 to 0 on the NRS). However, he had to apply the baclofen $5 \%$ cream 2-4 times a night, thus the duration of the analgesic effect was short: between 2 and 4 hours.

Phenytoin 5\% cream was added to the baclofen $5 \%$ cream on 30 May 2016. After the application of the combination cream, the patient had no aggravation of pain; instead he experienced direct pain relief and the prolongation of pain relief for 10 hours (the whole night). Most of the nights the patient only needed to apply the combination cream once before going to bed. His quality of sleep improved considerably. The amount per application was only 0.1 gram, which was applied on the big toe.

\section{Case 2: Small fiber neuropathy}

A 63-year-old male patient suffered during 1 year from neuropathic pain in both feet, distal parts of legs and hands due to small fiber neuropathy (SFN). This was confirmed by the neurologist based on the clinical examination via scoring on the small fiber neuropathy score: 51 points and by a positive warm water wrinkle test. The neurologist reported a 'very disabling small fiber neuropathy'. Secondary diagnoses were lupus erythematodes, sarcoidosis and lumbar disc pathology. Previous analgesic use, as prescribed by the neurologist, was pregabalin $525 \mathrm{mg}$ daily and oxycodone $10 \mathrm{mg}$ as needed. Further medications were tiotropium, fenprocoumon, tamsulosine, rosuvastatine, pantoprazol, levocetirizine, a calcium formulation and lisinopril. The main pain locations were the feet, distal part legs, distal part arms and hands, and the main pain characteristics were burning and stinging. Baseline NRS before treatment was 8-9. We prescribed phenytoin $10 \%$ cream, and the amount per application used by the patient was quite high: 1 tube of 30 grams in 4-5 days, thus 6.7 grams
Austin J Anesthesia and Analgesia - Volume 5 Issue 2 - 2017 ISSN : 2381-893X | www.austinpublishing group.com

Keppel Hesselink et al. (C) All rights are reserved
Citation: Keppel Hesselink JM and Kopsky DJ. Phenytoin Cream Reduces Pain and Improves Sleep in Patients Suffering from Neuropathic Pain. Austin J Anesthesia and Analgesia. 2017; 5(2): 1061. 


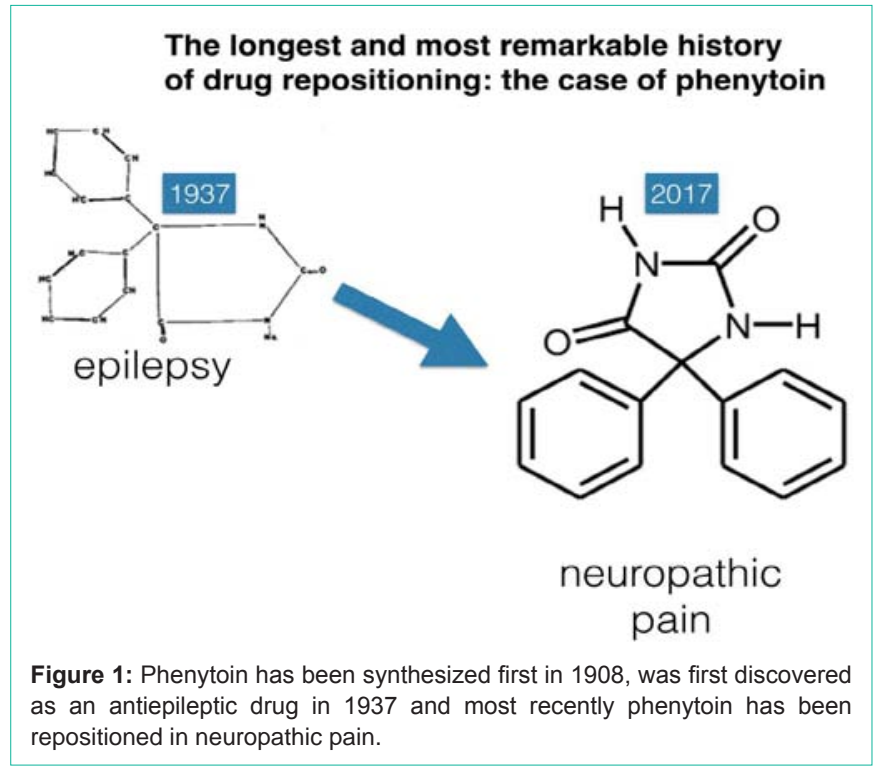

daily. The onset of analgesia as reported by the patient was fast, around 10 minutes. The pain reduction after application was around $60 \%$ to $70 \%$. The patient applied the cream once daily, beginning in February 2017 and ongoing. The duration of analgesic effect was 20 hours. In an email (March 2017) the patient stated:

1. Pain is reduced after approximately $5 / 10$ minutes following application of cream.

2. The analgesic effect lasts about 20 hours and there is almost no need to apply extra cream.

3. Sleep is $60 / 70 \%$ improved, before application I awoke due to pain 4 out of 5 nights.

4. My entire left leg was painful when I would sleep in a wrong position, and had to change position in bed, and even rise, but after cream application I can stay in bed and the pain is very much bearable.

5. My stability is much improved and I can now walk a bit further. Tingling is also less.

No plasma levels of phenytoin were found after 25 days of application once daily (6.7 grams per application). The last application before plasma sampling was 2.5 hours in order to measure at T-max time.

\section{Case 3: Decompression surgery}

A 58-year-old female was suffering from central neuropathic pain which had worsened since a decompression cyst Th12/L1 operation in 2005. The medical history consists of a hernia nucleus pulposus L5/S1 operation at the left side 30 years ago; a skiing accident in 1995 had led to a fracture of L1; and a cystic change of the conus medullar is was followed by two operations, which subsequently led to the removal of the osteo synthesis material 25 years ago. In 2006 a tethered cord L5/S1 operation followed; in 2010 a re-exploration due to a cyst; in 2011 a further exploration of the cyst; and in 2013 the placement of a syringosubdural drain inconus and scar resection. Given this complicated history and the neuropathic pain occurring after spinal surgery, the patient could be diagnosed as suffering from a Failed Back Surgery Syndrome (FBSS), a frequently observed troublesome and difficult to treat complication [12].

The main pain locations were feet and calves, and the pain characteristics mainly painful cold, tingling and cramps. Before application, the pain score was 7 on the NRS for cramps/cramping pain and 5 on the NRS for tingling. On the DN-4, a screenings questionnaire for neuropathic pain, she scored 8 out of 10 .

Initially we started treatment with amitriptyline 5\% cream without any effects, we then switched to baclofen $5 \%$ cream as we had experienced good effects of such cream in cramping pain due to central or peripheral origin.

The baclofen $5 \%$ cream had only a slight effect on the cramps and was substituted by phenytoin sodium 5\% cream on 3 September 2014. The phenytoin sodium 5\% cream, however, diminished the cramps completely. The phenytoin sodium $5 \%$ cream did not have any effect on the tingling. The cramps interfered with her sleep considerably (7 on the NRS). After the application of phenytoin sodium $5 \%$ cream, she no longer experienced any interference of her sleep ( 0 on the NRS).

The amount of phenytoin per application was 1.2 grams and she applied the cream twice daily. The onset of action was remarkably fast, just one minute and the cramps and pain were diminished completely to a 0 on the NRS. The duration of analgesic effect was 3 hours.

\section{Case 4: Diabetes mellitus neuropathy}

A 56-year-old male suffering from diabetes mellitus type II since April 2016 experienced neuropathic pain in both feet, with the pain characteristics of burning, tingling, and pins and needles, all in the same area as the numbness. He scored his pain as 8 on the NRS. His previous analgesic use was duloxetine $60 \mathrm{mg}$ daily. His medication at the time was pregabalin $450 \mathrm{mg}$ daily, metformin $500 \mathrm{mg}$ twice daily, atorvastatine $40 \mathrm{mg}$ once daily, short-acting insulin 4/4/6 units, and long-acting insulin 28 units.

We prescribed phenytoin $10 \%$ cream, and the patient applied 2 fingertip units to both feet. The patient reported an onset of action of 5 minutes, and pain was reduced by more than $50 \%$ to an NRS score of 3. The duration of analgesic effect was 8 hours. The patient applied the cream 2-3 times daily, and has used the cream for 4 weeks onwards.

The most intense pain was during night, with a pain score of 9-10 on the NRS, and the patient was unable to sleep. After applying the phenytoin $10 \%$ cream, he reported that he was able to sleep again and that the pain was considerably less.

\section{Discussion}

The above described cases all support the positive impact of topically applied phenytoin cream on pain and sleep quality as perceived by the patient. The effect is clearly a peripheral one, as we could not detect any plasma levels in a cohort of 16 patients.

Most of the patients we see come as referrals for second or third line therapy and have been multi-resistant to treatment according to general guidelines for the treatment of neuropathic pain. We have seen a number of such patients who do not respond to a selection of our creams, based on ketamine, amitriptyline or clonidine. However, some of these patients could turn into partial or full responders after 
switching to phenytoin $10 \%$ cream, or after adding such cream to the first prescribed topical analgesic. We have chosen a particular cream base based on its pharmaceutical properties of not being sticky or smelly, as many patients indicate is the case for pluronic organogel (PLO) gel. Moreover, this base cream enables us to mix various analgesics and combine those with phenytoin. However, there are always patients who remain non-responders to topical treatment. Based on non-blinded data, we feel that most responders suffer from pain due to diabetic neuropathy, small fiber neuropathy, CIPN and CIAP. The indications mentioned all support a peripheral mechanism of action, and elsewhere we have elaborated this mechanism [6]. Basically, phenytoin as a broad acting channel blocker with antiinflammatory and neuroprotective properties might influence 3 different cross-talking targets in the skin: the nociceptors, the keratinocytes and the immune-competent cells. All of these cellular elements contain sodium channels. Therefore, phenytoin would exert its analgesic effects by down regulating the peripheral wind-up phenomena based on cross-talk between these elements.

Phenytoin could be a new tool in our armamentarium of topical analgesics and should be considered more often as it does not induce intolerable side effects. We are currently preparing a series of blinded $\mathrm{N}$-of-1 studies, as well as a double-blind placebo controlled study, to further explore the safety and efficacy of this topical approach based on phenytoin.

\section{Conflict of Interest}

Authors are patent holders of two patents related to the topical formulations of phenytoin in the treatment of pain: 1) Topical phenytoin for the use in the treatment of peripheral neuropathic pain and 2) Topical pharmaceutical composition containing phenytoin, a co-analgesic for the treatment of chronic pain.

\section{References}

1. Keppel Hesselink JM and Kopsky DJ. Phenytoin: 80 years young, from epilepsy to breast cancer, a remarkable molecule with multiple modes of action. J Neurol. 2017; 264: 1617-1621.

2. Keppel Hesselink JM. Amantadine and phenytoin: Patent protected cases of drug repositioning. Clin Invest. 2017; 7: 11-16.

3. Keppel Hesselink, JM. Phenytoin: A step by step insight into its multiple mechanisms of action- 80 years of mechanistic studies in neuro pharmacology. J Neurol. 2017.

4. Keppel Hesselink JM and Kopsky DJ. Phenytoin: Neuroprotection or neurotoxicity? Neurol Sci. 2017; 38: 1137-1141.

5. Kopsky DJ and Keppel Hesselink JM. Phenytoin in topical formulations augments pain reduction of other topically applied analgesics in the treatment of trigeminal neuralgia. Journal of Clinical Anesthesia. 2017; 38: 154-155.

6. Kopsky DJ and Keppel Hesselink JM. Topical phenytoin for the treatment of neuropathic pain. J Pain Res. 2017.

7. Keppel Hesselink JM and Kopsky DJ. Topical phenytoin cream reduces burning pain due to small fiber neuropathy in sarcoidosis. J Anesth Pain Med. 2017; $2: 1-3$.

8. Keppel Hesselink JM and Kopsky JD. Topical phenytoin in neuralgic pain peripheral modulation of central sensitization: Two case reports. J Pain Relief. 2017.

9. Ojeda B, Salazar A, Calahorro MJ, Dueñas M, Mico JA, de Sola H, et al. Understanding the different relationships between mood and sleep disorders in several groups of non-oncological patients with chronic pain. Curr Med Res Opin. 2017.

10. Dogru Huzmeli E and Melek I. Neuropathic pain's biopsychosocial effects. Neurol Sci. 2017; 38: 1993-1997.

11. Melikoglu MA and Celik A. Does Neuropathic Pain Affect the Quality of Sleep? Eurasian J Med. 2017; 49: 40-43.

12. Cho JH, Jae Hyup Lee, Kwang-Sup Song, and Jae-Young Hong. Neuropathic Pain after Spinal Surgery. Asian Spine J. 2017; 11: 642-652.
Austin J Anesthesia and Analgesia - Volume 5 Issue 2 - 2017 ISSN : 2381-893X | www.austinpublishing group.com Keppel Hesselink et al. (C) All rights are reserved
Citation: Keppel Hesselink JM and Kopsky DJ. Phenytoin Cream Reduces Pain and Improves Sleep in Patients Suffering from Neuropathic Pain. Austin J Anesthesia and Analgesia. 2017; 5(2): 1061. 